
\title{
3 Research Soure \\ A modified SIR model for the COVID-19 epidemic in China
}

Jia-Le Wang

East China University of Technology

Yang Liu

East China University of Technology

Xu-Sheng Liu

East China University of Technology

Ke-Ming Shen ( $\square$ shen_keming.ecut@hotmail.com )

East China University of Technology

\section{Research Article}

Keywords: non-extensive, COVID-19, SIR model

Posted Date: May 26th, 2021

DOl: https://doi.org/10.21203/rs.3.rs-556631/v1

License: (c) (1) This work is licensed under a Creative Commons Attribution 4.0 International License. Read Full License

Version of Record: A version of this preprint was published at Journal of Physics: Conference Series on January 1st, 2022. See the published version at https://doi.org/10.1088/1742-6596/2148/1/012002. 


\title{
A modified SIR model for the COVID-19 epidemic in China
}

\author{
Jia-Le Wang, Yang Liu, Xu-Sheng Liu, and Ke-Ming Shen* \\ School of Science, East China University of Technology, Nanchang 330013, China
}

(Dated: May 24, 2021)

\begin{abstract}
The analysis of the coronavirus disease 2019 (COVID-19) is of great importance to deeply understand the dynamics of this coronavirus spread. Based on the complexity of it, a modified susceptibleinfected-removed (SIR) model is applied to analyze the time dependence of active and hospitalized cases in China. The time evolution of the virus spread in different provinces was adequately modeled. Changeable parameters among them have been obtained and turned to be not naively independent with each other. The non-extensive parameter was found to be strongly connected with the freedom of systems. Taken into the prevention and treatment of disease, more measures by the government lead to higher values of it.
\end{abstract}

\section{INTRODUCTION}

The coronavirus disease 2019 (COVID-19) has been a kind of global public health emergency [1] since it occurred in December 2019 in Wuhan, the capital of Hubei Province in China. The outbreak subsequently spread rapidly throughout China and elsewhere. More and more intervention strategies were implemented in China in order to contain this outbreak. It has caused significant disruption to the social and economic structure. Nevertheless, it is still unknown whether these policies have had an impact and how long they were able to remain there. On the other hand, the case abroad developed worse, which has reversely affected the epidemic progression in China then. It is thus critical to assess the effects of these control measures on the development of COVID19.

Nowadays in the market there have already existed plenty of models, for example, susceptible-infectedremoved (SIR), susceptible-infected-susceptible (SIS), and susceptible-exposed-infected-removed (SEIR) models, which have been proposed to predict the epidemic progression. However, all of these mathematical models of infectious disease shared some of ideal hypotheses: a) the reproduction number $R$ is often proposed to be exponential which is coming from the classical BoltzmannGibbs (BG) statistics; b) the internal interactions are always neglected when considering the propagation of disease, such as the normal human contacts in daily life; c) the total number is recognized as a constant, namely the system is a closed one; d) etc.

Though a large number of works [2-5] have made it possible to research on the development of COVID-19 within the previous models quantitatively, there still remains uncertainty in the theoretical framework, especially for the influences of so many control measures by our government and else. Consequently, this work would like to modify the simple SIR model in order to study the COVID-19 epidemic in China with respect to the nonextensive statistics. The non-extensive statistics, firstly

*Electronic address: shen_keming.ecut@hotmail.com proposed in [6] has attracted lots attention and discussions [7]. Instead of the normal exponential function, a generalized $q$-exponential function, defined as $[6,7]$,

$$
\exp _{q}(x):=[1+(1-q) x]^{\frac{1}{1-q}},
$$

was introduced to deal with the problems for the appearance of critical fluctuations due to interactions or non-ideal cases. One non-extensive parameter $q$ is to account for all possible factors violating assumptions of the usual BG cases. Its inverse function is also listed[6, 7],

$$
\ln _{q}(x):=\frac{x^{1-q}-1}{1-q}
$$

Both of them return to the usual exponential and logarithm function with $q \rightarrow 1$.

The purpose of this paper is to clarify the nonextensive effects on the fitting parameters of the modified SIR model for the COVID-19 epidemic. We focus on the situations in some provinces in China, where both similarities and differences appear. For instance, we have analyzed the situations in some coastal provinces, tierone-provinces, inland provinces and so on. In order to research on the effects of import cases from abroad, the situations in Taiwan and Hong Kong are also well studied within our model.

This paper is organized as follows. In Section II we introduce the theoretical framework, where the nonextensive $q$-SIR model is stated as well as the normal SIR one. Their comparisons are also explored afterwards. The consequences for various disease spreading situations in different provinces are exhibited in Section III; more detailed discussions on the results are also contained. Section IV is our brief summary and outlook.

\section{THEORETICAL FRAMEWORK}

As a classical compartmental model in epidemiology, the SIR model was created in 1927 [8] in which a fixed population was considered with only three compartments: 
1. $S(t)$, susceptible, is used to represent the individuals not yet infected with the disease at time $t$, or those susceptible to the disease of the population.

2. $I(t)$, infected, denotes the individuals of the population who have been infected with the disease and are capable of spreading the disease to those in the susceptible category.

3. $R(t)$, recovered, is the compartment used for the individuals of the population who have been infected and then removed from the disease, either due to immunization or due to death. Those in this category are not able to be infected again or to transmit the infection to others.

Using them one can establish the SIR epidemic model without so-called vital dynamics (birth and death, sometimes called demography) described above as follows [912]:

$$
\begin{aligned}
& \frac{\mathrm{d} S(t)}{\mathrm{d} t}=-\beta I(t) S(t) \\
& \frac{\mathrm{d} I(t)}{\mathrm{d} t}=\beta I(t) S(t)-\gamma I(t) \\
& \frac{\mathrm{d} R(t)}{\mathrm{d} t}=\gamma I(t)
\end{aligned}
$$

where $\beta$ is the average number of contacts per person per time, multiplied by the probability of disease transmission in a contact between a susceptible and an infectious subject, and $\gamma I(t)$ is assumed to describe the transition rate between $I(t)$ and $R(t)$. The first differential equation is the fraction of those contacts between an infectious and susceptible individual which result in the susceptible person becoming infected. Worth to mention that in which there is no removal from the infectious compartment $(\gamma=0)$, the SIR model reduces to a very simple SI model, which has a logistic solution, in which every individual eventually becomes infected.

Considering the SIR system is closed:

$$
S(t)+I(t)+R(t)=N,
$$

it is possible to derive its analytic solution in implicit form [13]:

$$
I(t)=S_{0}+I_{0}-S(t)+\frac{1}{\sigma} \ln \frac{S(t)}{S_{0}}
$$

where $\sigma=\beta / \gamma$ is the basic reproduction number (also called basic reproduction ratio). $S_{0}$ and $I_{0}$ denote the initial numbers of, respectively, susceptible and infectious subjects. It is derived as the expected number of new infectious (sometimes called secondary infections) from a single infection in a population where all subjects are susceptible. [14, 15]

Note that the function, $F=\beta I$, in the above model, is recognized as the force of infection, which models the transition rate from the compartment of susceptible individuals to the compartment of infectious individuals.
However, for large classes of communicable diseases it is more realistic to consider a force of infection that does not linearly depend on the absolute number of infectious subjects. As for the stock of susceptible population, it has shown us that $S \approx N$ for most of cases due to the large population in SIR system. Therefore, these evolution equations need to be reconsidered.

Similar to the non-extensive statistics, the generalized evolution equations of COVID-19 are modified as follows:

$$
\begin{aligned}
& \frac{\mathrm{d} S(t)}{\mathrm{d} t}=0 \\
& \frac{\mathrm{d} I(t)}{\mathrm{d} t}=b I^{q}(t)-\gamma I(t) \\
& \frac{\mathrm{d} R(t)}{\mathrm{d} t}=\gamma I(t)
\end{aligned}
$$

whose solutions are listed as[*]:

$$
\begin{aligned}
S(t)= & N \\
I(t)= & A \cdot\{1-B \exp [-\gamma(1-q) t]\}^{\frac{1}{1-q}} \\
R(t)= & \frac{A}{\gamma(2-q)} \cdot \\
& \{1-B \exp [-\gamma(1-q) t]\}^{1+\frac{1}{1-q}}
\end{aligned}
$$

With such a $q$-power modified term of infectious individuals in Eq.(12), we then explore the time dependence of active and hospitalized cases in various kinds of provinces in China, typically for the number of infectious individuals. Worthy to mention that for such a distribution, there are already some other similar works appearing to describe satisfactorily the available data for COVID-19. For instance, C. Tsallis and U. Tirnakli [16] applied one $q$-statistical functional form in order to predict the peak$\mathrm{s}$ around the world. The numerical results will be shown in the next section.

\section{RESULTS AND DISCUSSION}

We start our discussions with presenting in Fig.1 the epidemic progression in several provinces in China from January 2020 to January 2021. Data are taken from [17]. According to the report of China CDC, January 12020 was considered as the 1st day of a contagion period, so we focused on these data sets. It indicates that all the year of 2020 there appeared almost ZERO new death individuals in China (seen as the green dots and lines in Fig.1), which nicely shows the effects caused by control strategies by

[*] More precisely, the exact solution of $I(t)$ should be $I(t)=\left(\frac{b}{\gamma}-\right.$ $\left.\exp \left[\gamma(1-q)\left(t_{0}-t\right)+\frac{1}{\gamma(q-1)} \ln \left(b-\gamma I_{0}^{1-q}\right)\right]\right)^{\frac{1}{1-q}}$. Similar for the distribution of $R(t)$. Without the loss of generality, we re-write them as above for simplicity. 

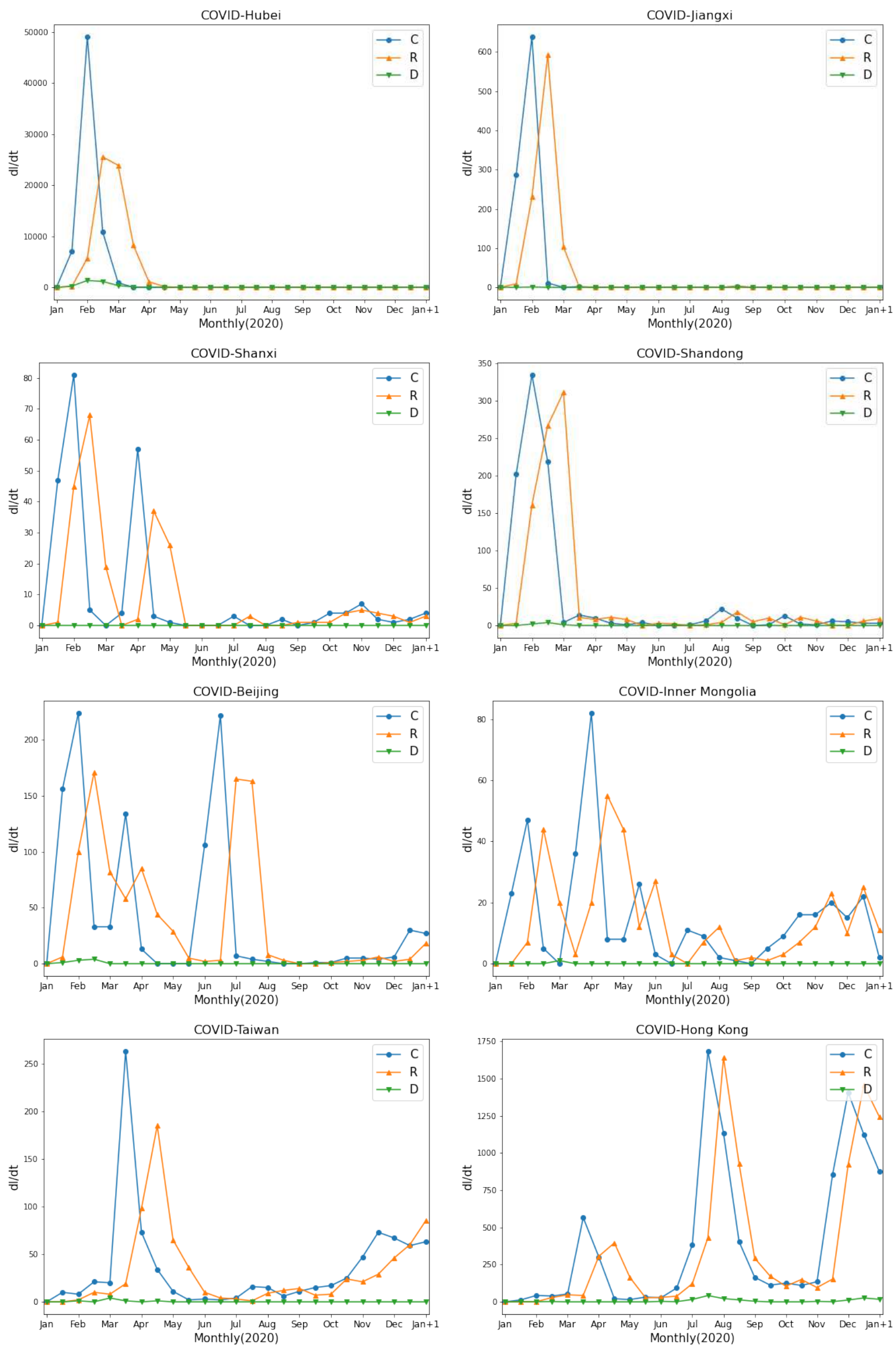

FIG. 1: Actual monthly data of Confirmed Infections $(C)$, Recovered Cases $(R)$ and Dead Individuals $(D)$ for several provinces (Hubei, Jiangxi, Shanxi, Shandong, Beijing, Inner Mongolia, Taiwan and Hong Kong, respectively). 
the government. While both the confirmed and recovered infections vary heavily among all the provincial data.

For the upper two plots, they show the epidemic progression for provinces like Hubei and Jiangxi. From them we could see that all data of infections (both $C$ and $R$ ) vanish from around May of 2020. This is largely due to the effectiveness of quarantine and control measures in China, especially the lock-down of Wuhan city from January 23, 2020 to April 8, 2020.

The cases in Shanxi and Shandong are distributing similarly as above but some exceptions where a slight outbreak happened there. This could be obviously seen as in the next two panels. However, there are still many provinces or cities in which the COVID-19 still exists and even arises a secondary infection. Because of the frequent contacts and interchanges with outside, the size of system in cities like Beijing and Inner Mongolia is very huge as well as its complexity. That is to say, they are more like open systems.

On the other hand, it is instructive to plot the distributions in Hong Kong and Taiwan, where the effects from abroad cannot be neglected indeed. The lower two panels show how the imported cases work on the spread of this contagious disease.

In virtue of it the validity of approximation, $S_{0} \approx N$, is nicely supported by real data in China [18], and the development of recovered individuals in SIR model strongly relies on the case of infectious ones, in this work we shall focus on the possible interpretations of spread of infections within the non-extensive effect. Furthermore, all the evolutionary tendency of infectious individuals are to be fitted by the distribution:

$$
\begin{aligned}
I(t) & =A \cdot[1-B \exp [-\gamma(1-q) t]]^{\frac{1}{1-q}} \\
& =a \cdot[1-b \cdot \exp (-c t)]^{d}
\end{aligned}
$$

Worthy to note that the large values of time $t$ could recover an exponential function whereas it leads to a Tsallis $q$-exponential distribution, cf. Eq.(1) when $t \rightarrow 0$. On the other hand, $\gamma$ still gives out the information on the average time for which an individual is infectious. The non-extensive parameter $q$ tells us the departure from the ideal situation, in which the distribution behaves as an exponential function.

In our programs the fitting function has been simplified as shown in Eq.(14) with the new parameters, $c$ and $d$ being $q$-dependent. By using it we analyzed the monthly spread of infections from January of 2020 to January of 2021. In order to better understand the mechanism of the infectious numbers, we summed up all the previous new confirmed cases as the number of infections by then. All the annual situation was analyzed since we would like to see the connections or differences among different provinces.

In Table I all the fitting parameters in Eq.(14) are tabulated for various kinds of provincial data. Almost the same values of normalization constant, $b$, indeed tell us that there is no big difference among these provinces
TABLE I: The values of fitting parameters in Eq.(14) in the modified SIR model for different provinces:

\begin{tabular}{l|l|c|c|c}
\hline Provinces & $a$ & $b$ & $c$ & $d$ \\
\hline Hubei & 100000 & 1.010 & 1.023 & 0.20 \\
Jiangxi & 1500 & 1.009 & 0.886 & 0.21 \\
Shanxi & 290 & 1.009 & 0.851 & 0.16 \\
Shandong & 1150 & 1.009 & 0.892 & 0.16 \\
Beijing & 1050 & 1.101 & 12.394 & 0.92 \\
Inner Mongolia & 550 & 1.015 & 4.040 & 0.92 \\
Taiwan & 50 & 0.456 & -1.708 & -2.4 \\
Hong Kong & 80 & 0.653 & -1.141 & -2.4 \\
\hline
\end{tabular}

except for Taiwan and Hong Kong, which were largely affected by the imported cases. The weak fluctuation appeared in the fittings for Beijing's infectious distributions. This could be nicely explained by its speciality of tier-one-city.

On the other hand, as shown in Eq.(14), the production of parameters $c$ and $d$ explores the information of the average time, $\gamma$. It varies between the normal and high-risk areas for COVID-19 in China. As for the nonextensive parameter, $q=1-1 / d$, there was no big fluctuation among most of provincial data as well, especially for the relatively close provinces such as Beijing and Inner Mongolia. This indicates the common spread mechanism among most parts in China. While we cannot neglect the big divergence for the cases in Taiwan and Hong Kong.

At the same time, one can easily observe an obvious connection between the values of fitting parameter $a$ and the infectious peaks in all provinces. For example, the situation in Hubei was nearly the most serious in China at the beginning of the year 2020 and even the government took the control measures by locking down some cities in Hubei for some time. This could be well seen by the maximum number of parameter, $a=100000$, of all.

In order to illustrate the fittings of this modified SIR model for the COVID-19 pandemics in China more clearly, fitting results are plotted in figures 2, 3, 4 and 5. Our model is proved to be an alternative tool to investigate the spread of infectious individuals for COVID-19 in China.

Specifically, Fig.2 lists the fittings on the monthly evolution of infectious individuals for the provinces of Hubei and Jiangxi. We could see that the number of infections reached an almost constant from March of 2020, which is shown in our fit values (seen as the red lines). No doubt that the shape looks similar since the Chinese government took all the control measures for the whole country. The numerical difference at the peak lights upon their own outbreaks at the initial period of COVID-19 in China, for example the case was much better in Jiangxi at that time.

A little difference happened to the cases for Shanxi and 
Shandong, as shown in Fig.3. As reported by the local governments, there existed some few infectious cases at the end of autumn in 2020, which has been proved to be the consequence of some people who were previously infected abroad and then returned home. This leads to a better fitting consistency where there were more nonextensive interactions in the system.

As expected, the spread in the tier-one-city, Beijing, is more complicated. Because of the frequent connection$\mathrm{s}$ with outside, it is much more easily to see that the non-extensive effects cannot be neglected. As a result, our model is more proper to describe their disease development. In Fig.4 the distribution cf. Eq.(14) nicely coincides with the original values of infectious individuals. Similar cases happened to the provinces like Inner Mongolia and Xinjiang because of their special geographical locations.

Finally it is also important to explore the results for the cases in Taiwan and Hong Kong, where the imported factors are more inevitable. Moreover, most controlling measures taken in mainland of China were NOT well ap-
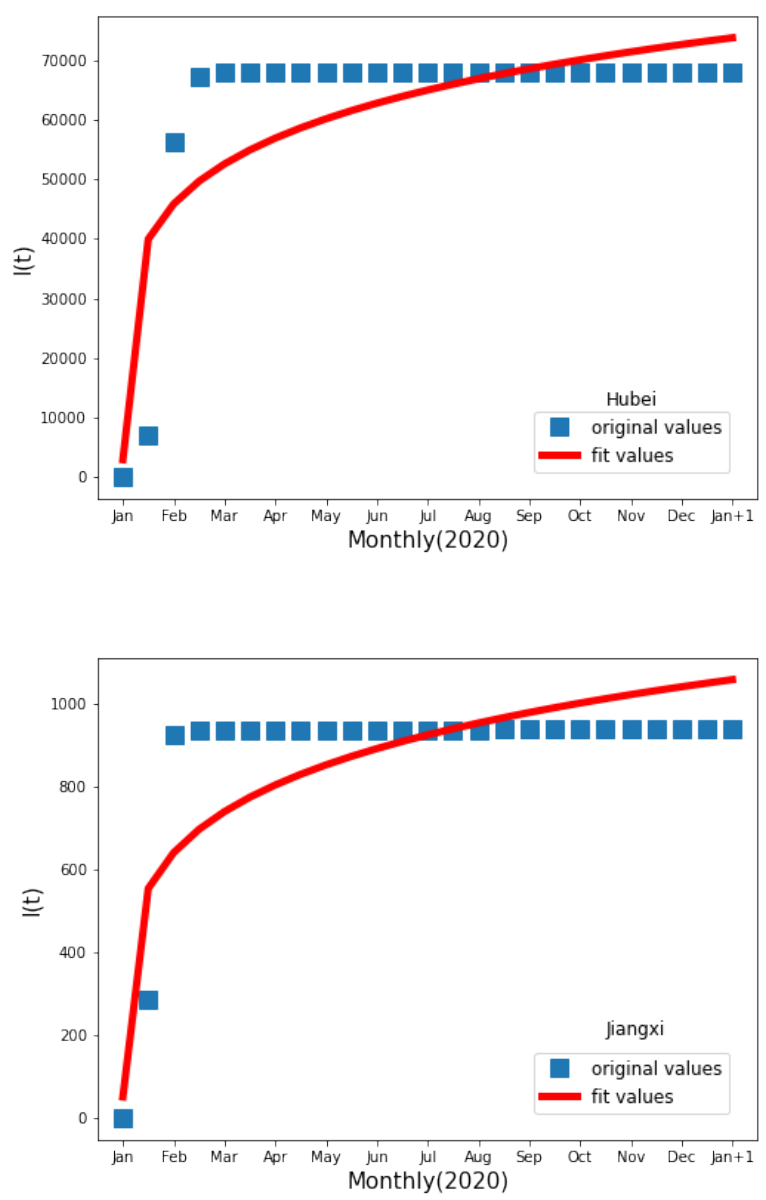

FIG. 2: Fittings on the monthly evolution of infectious individuals for the provinces of Hubei and Jiangxi.
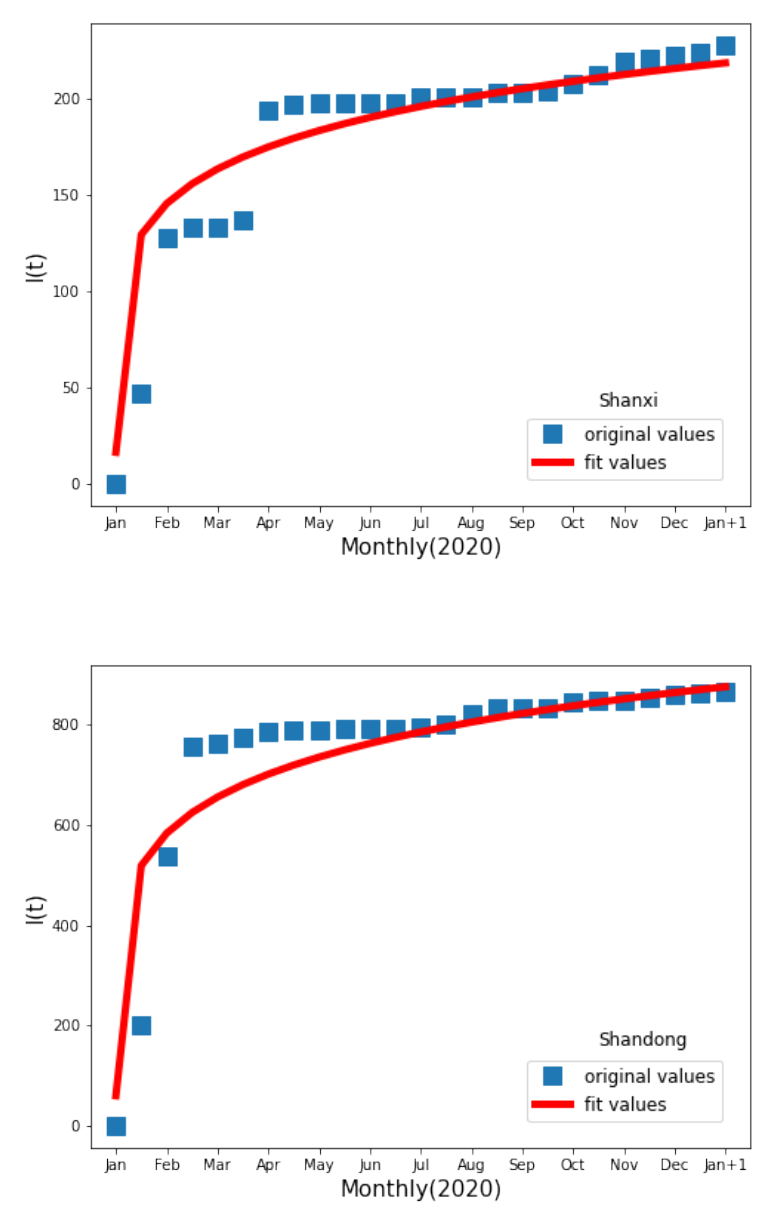

FIG. 3: Fittings on the monthly evolution of infectious individuals for the provinces of Shanxi and Shandong.

plied in these parts. Even when a secondary outbreak happened, the local government somehow neglected it. Thus the situation there became much worse at the end of 2020. Fig.5 agrees with the discussions above, that our modified SIR model is typically for the system which meets with more interferences from outside.

\section{SUMMARY AND OUTLOOK}

To summarize, we have calculated the non-extensive SIR model to account for the complexity of the epidemic spreads of the COVID-19 in China. Especially the Chinese government has taken many emergency actions in order to help reduce the contagion probability and cure the infectious individuals. On the other hand, the imported disease cases unavoidably affects the exploration of COVID-19 in China. All lead to it that the system for this epidemic transmission should be considered within the non-extensive application rather than the classical ideal one. By the $q$-version we have modified the original 

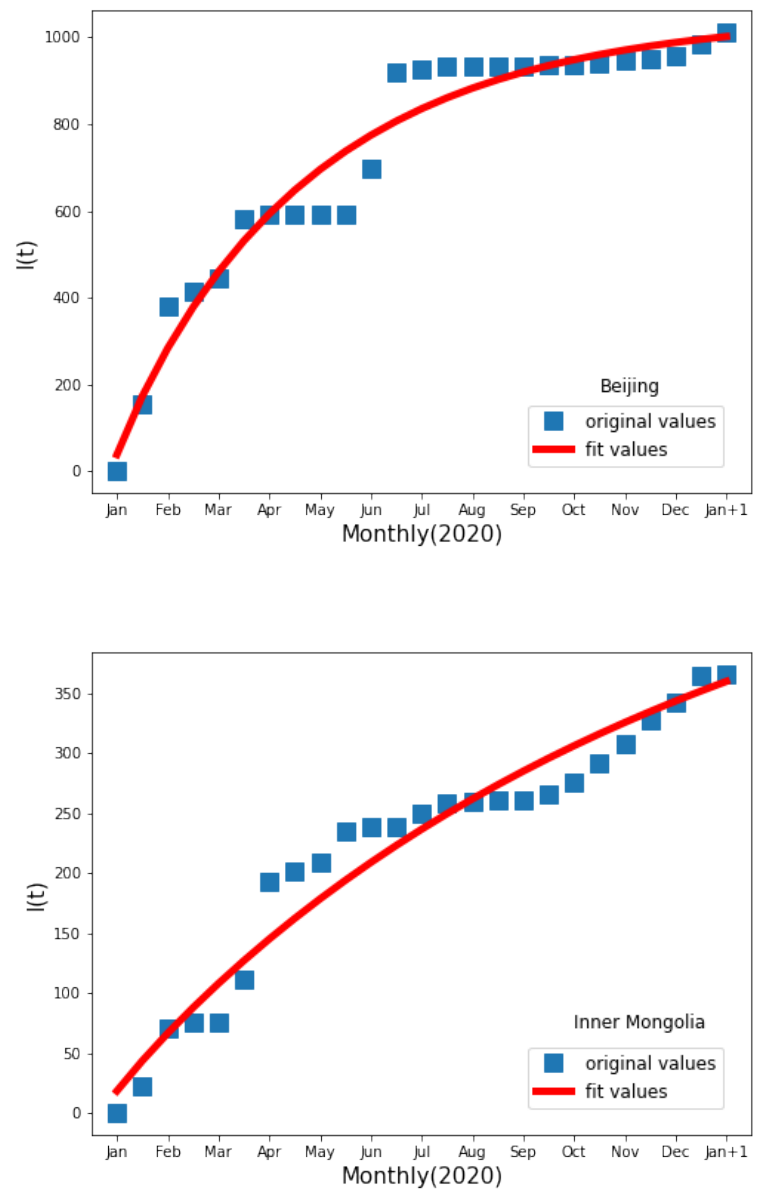

FIG. 4: Fittings on the monthly evolution of infectious individuals for Beijing and Inner Mongolia.

SIR model to investigate the time evolution of infectious individuals in different provinces in China.

It is of importance to research on the fittings within our modified SIR model for the various provincial data sets. All the corresponding fitting parameters illuminate that the non-extensive application works well on the spread of infectious individuals in mainland of China. Compared with the cases in several typical provinces, such as Hubei, Beijing and others, we indeed found out the common properties among different places in mainland of China. This is due to the fact that all the effective actions were taken in all corresponding cities. While it is worthy to note the cases in Taiwan and Hong Kong, for which the system prefers to an ideal one since nearly no effective measures were taken or no enough notations were caused by the local government at the initial time.

Last not the least, a complete understanding of the physics of non-extensive parameters in the study of COVID-19 should be solved in general context. In this paper we have tried to illuminate it from the comparisons among various fittings of provincial data sets in China.
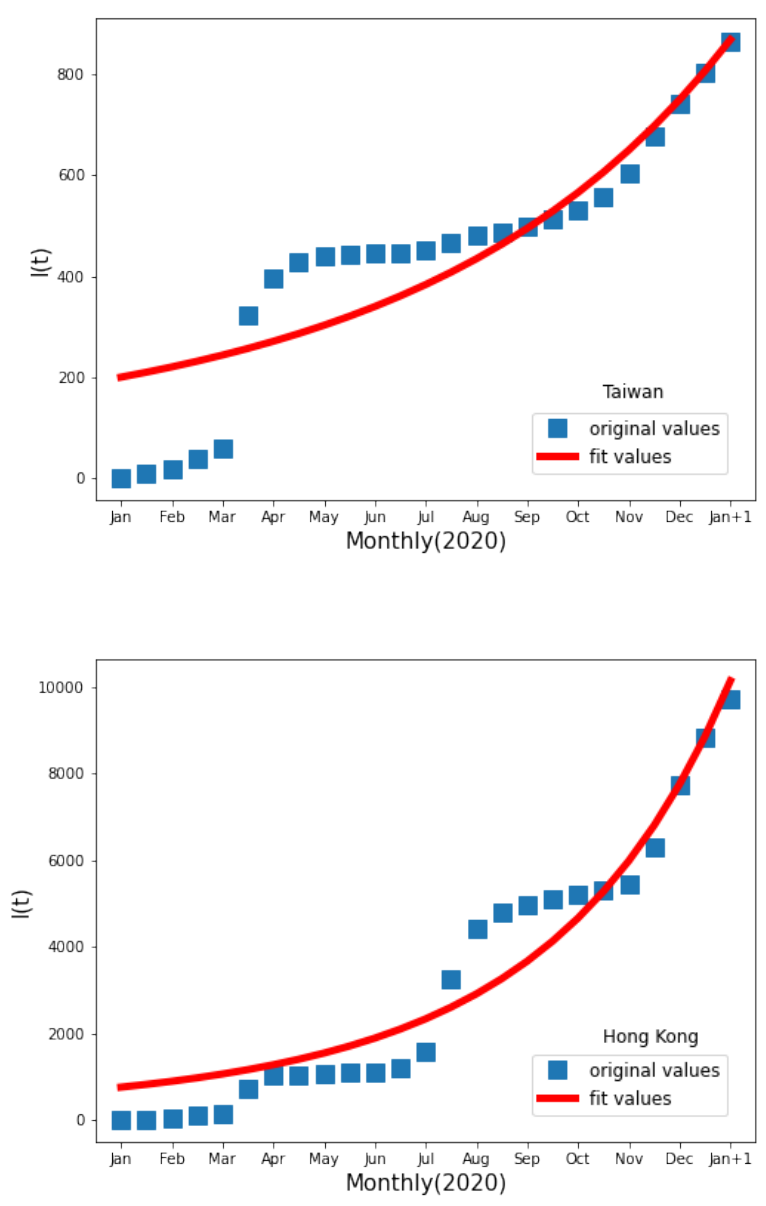

FIG. 5: Fittings on the monthly evolution of infectious individuals for Taiwan and Hong Kong.

Our model, on the other hand, successfully provides another method to investigate on problems in the scientific researches on the epidemic disease such as COVID-19. Meanwhile, our researches could deeply help understand the physical explanation of the Tsallis non-extensive parameter $q$, which is also what we will pay attention to in the future.

\section{Acknowledgments}

This research is partly supported by the funding for the Doctoral Research of ECUT Nos. DHBK2019211 and DHBK2019200 and by NSFC under Grant No. 11947020.
[1] WHO: Coronavirus disease 2019 (COVID-19): situation report, 51 (2020).
[2] Z. Yang, et. al., J. Thorac. Dis 2020; 12(3): 165-174 
[3] Teles P. Bull World Health Organ. E-pub: 7 April 2020.

[4] Z. Ahmad, et. al., Scientific. Reports (2020) 10:22268.

[5] M. Radha and S. Balamuralitharan, Adv. in Diff. Equa. (2020) 2020:523.

[6] C. Tsallis, J. Stat. Phys. 52, 479 (1988).

[7] Lists of many applications of non-extensive statistics are available at http://tsallis.cat.cbpf.br/biblio.htm.

[8] W. O. Kermack and A. G. McKendrick, Bulletin of Mathematical Biology. 53 (1991) 3355.

[9] E. Kenah and J. M. Robins, J. Theor. Biol. 249, 706 (2007).

[10] E. Kenah and J. M. Robins, Phys. Rev. E 76, 036113 (2007).

[11] S. Cauchemez and N. M. Ferguson, J. R. Soc., Interface 5, 885 (2008).

[12] Yong Tao, Phys. Rev. E 102, 032136 (2020).
[13] T. Harko, Lobo, S. N. Francisco and M. K. Mak, Applied Mathematics and Computation. 236 (2014) 184194.

[14] Bailey, Norman T. J. (1975). The mathematical theory of infectious diseases and its applications (2nd ed.). London: Griffin. ISBN 0-85264-231-8.

[15] Sonia Altizer and Nunn, Charles (2006). Infectious diseases in primates: behavior, ecology and evolution. Oxford Series in Ecology and Evolution. Oxford [Oxfordshire]: Oxford University Press.

[16] C. Tsallis and U. Tirnakli, Fron. Phys. 8: (2020) 217.

[17] National Health Commission of the People's Republic of China, The latest information on the infections of COVID-19 [EB/OL], http://www.nhc.gov.cn/xcs/ yqtb/list_gzbd.shtml

[18] Yong Tao, Phys. Rev. E 102, 032136 (2020). 